\title{
Eric Weil e la «fine della storia»
}

\author{
Eric Weil e o "fim da história"
}

\section{Eric Weil and "The End of History"}

DOI: https://doi.org/10.20873/rpv6n1-93

\author{
Edoardo Raimondi \\ Orcid: https://orcid.org/0000-0001-6580-1907 \\ Email: edoardoraimondi1@gmail.com
}

\section{Sommario}

Intento di questo contributo è mostrare come Eric Weil (1904-1977) abbia ricompreso la famosa tesi della "fine della storia", elaborata da Alexandre Kojève (1902-1968) sin dagli anni '30, proponendone una nuova versione di carattere essenzialmente "kantiano posthegeliano": un elemento teorico fondamentale dell'iter storico-filosofico weiliano, che sarebbe emerso chiaramente dagli studi specifici su Kant a cui Weil si dedicò in modo puntuale dagli inizi degli anni '60. Si vedrà, quindi, come i risvolti "pratici" di tale ricomprensione abbiano di fatto indicato la strada per la costituzione di una nuova filosofia politica, eminentemente critica e antidogmatica.

\section{Parole-chiave}

Alexandre Kojève. Eric Weil. Dialetica Hegeliana. Fine della Storia. Modernità. Postmodernità.

\section{Resumo}

O artigo visa mostrar como Eric Weil (1904-1977) repensou a famosa tese do "fim da história", elaborada por Alexandre Kojève (1902-1968) desde os anos de 1930, propondo uma nova versão de caráter essencialmente "kantiano pós-hegeliano": um elemento teórico fundamental do inter histórico-filosófico weiliano, que emergiria claramente dos estudos específicos sobre Kant aos quais Weil se dedicou de modo pontual a partir da década de 1960. Veremos, portanto, como as reviravoltas "práticas" dessa nova compreensão tenham de fato indicado a estrada para a constituição de uma nova filosofia política, eminentemente crítica e antidogmática.

\section{Palavras-chave}

Alexandre Kojève. Eric Weil. Dialética Hegeliana. Fim da História. Modernidade. Pós-modernidade. 


\begin{abstract}
The aim of my article is to how Eric Weil (1904-1977) suggested a "Kantian-post-Hegelian" version of the famous thesis on the "End of History", elaborated by Alexandre Kojève (1902-1968) from the 1930s. We'll see how this aspect of the Weilian thought, emerged clearly from the specific studies on Kant that Weil developed since the early 1960s. Therefore, will be clear how the "practical" implications of this Weilian reunderstanding indicated the way a new political philosophy, eminently critical and anti-dogmatic.
\end{abstract}

\title{
Keywords
}

Alexandre Kojève. Eric Weil. Hegelian Dialectic. End of History. Modernity. Postmodernity.

\section{Alexandre Kojève, Eric Weil e la «Hegel renaissance»}

Sappiamo che i famosi seminari hegeliani all'École Pratique d'Hautes Études, affidati da Alexandre Koyré a Kojève nel 1933, segnarono di certo una data cruciale per la Hegel renaissance francese, come è noto iniziata agli albori degli anni Venti del secolo scorso ${ }^{1}$. La teologia kierkegaardiana di Jean Wahl, il platonismo di Koyré, la fenomenologia husserliana, le tesi principali dell'Heidegger di Sein und Zeit, la reazione al riduzionismo epistemologico e i nuovi interessi surrealisti per la psicanalisi, così come la scoperta della filosofia del giovane Marx, furono gli ingredienti essenziali che permisero a Kojève di rileggere la Fenomenologia dello spirito di Hegel in chiave antropologica, fenomenologica ed esistenzialista ${ }^{2}$. Ne conseguì una rivisitazione dell'hegelismo in ottica dualistica e soggettivistica, così come uno stravolgimento dell'oggettività della dialettica e del significato della hegeliana negazione determinata. Il comprovato tentativo di Koyré di ricondurre il Geist hegeliano sulla terra attraverso la fenomenologia husserliano-heideggeriana trovò così terreno fertile durante $\mathrm{i}$ seminari a Hautes Études, attraverso cui Kojève cercò di dimostrare ciò che Koyré, durante i

\footnotetext{
${ }^{1}$ Per una ricostruzione di quel rinnovamento hegeliano, impossibile dimenticare le presentazioni complessive dei protagonisti e degli interpreti di quella stagione svolte in CANGUILHEM, 1948-49, p. 282-297; FETSCHER, 1953, p. 3-15; HYPPOLITE, 1971, p. 231-41; SALVADORI, 1974; BIEMEL, 1974, p. 643-55; CIAMPA, 1985, p. 129-148; NEGRI, 1987, p. 42-51; ROTH, 1988, p. 1-15; D'HONT, 1993, p. 49-53; JARCZYK; LABARRIÈRE, 1996, p. 17-36. Cfr. infine VALENTINI, 1958 - dove, in questo caso, si dà ampio rilievo al pensiero weiliano. Cfr. quindi VALENTINI, 2001, p. 271-343.

${ }^{2}$ A riguardo, si veda almeno AUFFRET, 1990; DATI, 1998, p. 91-126; FILONI, 2008; GEROULANOS, 2010; JARCZYK; LABARRIÈRE, 1996; PALMA, 2017; PIROTTE, 2005; ROTH, 1988, p. 83-146; SALVADORI, 1980, p. VII-XVIII; VEGETTI, 1999.
} 
primi anni Trenta, aveva solo ipotizzato: la famosa teoria della fin de l'histoire ${ }^{3}$. E così Kojève, facendo reagire su uno Hegel "fenomenologo" ed "esistenzialista” il pensiero di un giovane Marx ancora influenzato da Feuerbach (soprattutto quello dei Manoscritti economico-filosofici del 1844, pubblicati per la prima volta nel 1932), riuscì a concludere il radicale ateismo della filosofia hegeliana. Ne derivò una vera e propria eterodossia "hegelo-marxista" che alimentò una concezione teologica ed escatologica della storia in grado di giustificare laicamente la presunta realizzazione del paradiso terrestre, il trionfo eterno della forma dello stato moderno in quanto "stato universale e omogeno". Tuttavia, questa teologia laicizzata rimase inevitabilmente sospesa tra modernità e postmodernità, tra rivoluzione e reazione: non fu un caso che, soprattutto dopo gli anni '50, le tesi finali di Kojève sembrarono allontanarsi definitivamente da qualsiasi forma autentica di "hegelo-marxismo"4.

In tal senso Weil, uno degli uditori dei seminari kojèveiani, almeno fino agli anni '50 condivise chiaramente alcuni degli assunti del commento di Kojève a Hegel, in primis l'idea che il soggetto principale della Storia non potesse che essere l'uomo alienato, quel servo di hegeliana memoria in costante ricerca di riconoscimento e di soddisfazione concreta: la lotta rivoluzionaria per il riconoscimento di sé e la funzione del lavoro si confermavano le molle del divenire storico, così comprensibile come processo d'emancipazione universale messo in moto

\footnotetext{
${ }^{3}$ Cfr. KOYRÉ, 1972; KOJÈVE, 1996. D'ora in poi indicherò quest'opera con la sigla LFS.

${ }^{4}$ Fino all'anno di I pubblicazione dei seminari (1947), per spiegare l'evento della "fine della storia" (in quanto fine della "preistoria"), Kojève rimandava chiaramente al III libro del Capitale, facendolo così retroagire sullo Hegel della Fenomenologia e dello Stato napoleonico. In tal modo, la "fine hegeliana" poteva già coincidere con l'avvento del «Regno della libertà», in cui «gli uomini (riconoscendosi reciprocamente senza riserve), non lottano più e lavorano il meno possibile (dato che la Natura è stata definitivamente domata, cioè armonizzata con l'Uomo). Cfr. Il Capitale, libro terzo, capitolo XLVIII, fine del secondo capoverso del par. 3» (cfr. LFS, p. 541, nota 1). Tuttavia, come testimonia la famosa aggiunta alla nota sulla fin de l'histoire nella II edizione delle LFS (1968), fu lo stesso Kojève a prospettare una fine della storia in quanto termine del "propriamente umano" in senso peggiorativo, come avvento cioè degli «animali post-storici delle specie Homo sapiens (che vivranno nell'abbondanza e in piena sicurezza)» e che «saranno contenti in funzione del loro comportamento artistico, erotico e ludico, visto che, per definizione, essi se ne accontenteranno». Si spiega così perché il filosofo parlasse dell' «annientamento definitivo dell'Uomo propriamente detto»: ciò significava «la scomparsa definitiva del Discorso (Logos) umano in senso proprio. Gli animali della specie Homo sapiens», aggiungeva Kojève, "reagirebbero con riflessi condizionati a segnali acustici o mimici e così i loro cosiddetti "discorsi" sarebbero simili al presunto "linguaggio" delle api. Ciò che allora scomparirebbe non sarebbe soltanto la Filosofia e la ricerca della Saggezza discorsiva, ma anche questa stessa Saggezza. Infatti, non si avrebbe più, in questi animali post-storici, "conoscenza [discorsiva] del Mondo e di sé"». Cfr. $L F S$, p. 542, nota 1.
} 
dai protagonisti della modernità, quelle soggettività storiche spinte concretamente a esercitare la propria negatività ${ }^{5}$. Anche per Weil, quindi, il momento apicale di tale processo, come aveva affermato lo Hegel di Kojève, si era rivelato essere quello della rivoluzione francese, evento epocale che aveva portato a piena maturità lo sviluppo del mondo moderno attraverso l'affermazione politica del principio di uguaglianza universale tra gli uomini. Tuttavia, ciò non impedì a Weil di mettere in discussione sin da subito le conclusioni hegelo-kojèveiane, che avevano condotto a una vera e propria ideologia della fine: fine della storia e della filosofia che, con Hegel, si era trasformata in saggezza assoluta, avendo rivelato una volta per tutte la Verità incarnata dell'esistenza umana. Pur ammettendo con Kojève che nella Fenomenologia dello spirito si era effettivamente affermato il raggiungimento del fine universale della Storia - il compiuto riconoscimento di tutti e di ciascuno nel mondo reale - Weil sostenne che non era da quell'opera hegeliana che si doveva iniziare se si voleva comprendere davvero il sistema di Hegel. Prendendone piuttosto in considerazione gli scritti della maturità (quelli del sistema compiuto), Weil cercò di dimostrare come la filosofia della storia hegeliana non potesse esser ridotta a una "teologia laicizzata", elemento che, al contrario, tanto gli sviluppi

\footnotetext{
${ }^{5}$ Weil lo fece intendere chiaramente in uno dei suoi testi più significativi, Hegel e lo stato: cfr. WEIL, 1988, pp. 37152. In ogni caso, è cosa nota che ad assistere ai seminari di Kojève fu una platea decisamente d'eccezione. Lo ascoltarono, per diversi semestri accademici, anche George Bataille, Henry Corbin, Gaston Fessard, Jacques Lacan, Raymond Polin, Raymond Queneau, Maurice Merleau-Ponty, Weil, tra i più assidui, ma vi furono pure Raymond Aron, Robert Marjolin, Oliver Wormser, Leo Strauss. A questi nomi, attestati sull'annuario e sul registro delle iscrizioni della Scuola, vanno aggiunti sicuramente quelli di Jean Hyppolite, André Breton e Hannah Arendt. Cfr. in merito L. SICHIROLLO, 1997, p. 19; FILONI, 2013, p. X; l'elenco attestato dei partecipanti si trova in AUFFRET, 1990, p. 238, nota 1; e in ROTH, 1988, p. 225-27. Auffret ha notato che in questa lista manca il nome di Jean-Paul Sartre, «che conobbe il Seminario solo grazie all'articolo di Mesures» del 14 gennaio 1939 - articolo che poi sarebbe stato inserito nella raccolta delle lezioni con il titolo En guise d'introduction (e che riguarda il commento di Kojève alla sezione A del cap. IV della Phänomenologie: cfr. ibid). Sarte ne sarebbe rimasto «fortemente impressionato», come testimoniato da Simone de Beauvoir: cfr. a riguardo PIROTTE, 2005, p. 20. Tuttavia, si è anche ipotizzato che Sartre (come molti altri) avesse ascoltato di persona le tesi kojèveiane su Hegel, ipotesi avvalorata, per esempio, da Stefanos Geroulanos: «La lista dei partecipanti, pubblicata da Roth in Knowing and History [...], è di certo incompleta. Si dovrebbero aggiungere, infatti, gli uditori del Collegio di Sociologia dove Kojève diede spiegazione dei "concetti hegeliani" (coloro che presero parte a questa discussione furono Claude Levi-Strauss, Michel Leiris, Roger Caillois e altri habitué del Collegio, tra cui - cosa interessante - Walter Benjamin)» (GEROULANOS, 2010, p. 133, nota 6).
} 
dell'esistenzialismo francese quanto un certo marxismo ortodosso avevano già posto in nuce in modo differente 6 .

Il dualismo soggettivistico di Kojève, del resto, era in qualche modo riuscito a ricondurre il pensiero hegeliano a dettami teorici non solo di stampo kantiano e heideggeriano, ma ancor prima di matrice essenzialmente platonica (e cristiana). La ben nota teoria del desiderio di desiderio, scaturita illecitamente dalla lettura del IV capitolo della Fenomenologia dello spirito, mostrava così il risvolto "antropologico" di un dualismo fenomenologico e ontologico di matrice husserliana e heideggeriana, che già Koyré aveva utilizzato per rileggere Hegel. Ne derivò un soggettivismo essenzialmente astratto che finì per relegare la dialettica oggettiva di Hegel al solo ambito della temporalità umana. Insomma, in tale scenario interpretativo il pensiero del giovane Marx finiva per fondersi con quello di uno Hegel umanista ed esistenzialista, là dove si trattava di riaffermare i diritti della libertà umana e agente per scongiurare le teorie economiciste e riduzioniste della storia imposte dal dogma marxista ${ }^{7}$. Tenendo ben a mente tali

\footnotetext{
6 Proprio in Hegel e lo stato, Weil chiariva: «Tranne rare eccezioni, dunque, non abbiamo parlato né degli scritti giovanili né della Fenomenologia dello spirito, e abbiamo fondato la nostra interpretazione sulla Filosofia del diritto, utilizzando come sussidio l'Enciclopedia delle scienze filosofiche. I testi che non sono stati redatti personalmente da Hegel, in particolare le Aggiunte alla Filosofia del diritto che i curatori delle Opere complete hanno tratto dalle lezioni del loro maestro, le Lezioni sulla filosofia della storia e le Lezioni sulla storia della filosofia, ci hanno fornito solo chiarimenti e formulazioni, e unicamente quando lo consentiva una testimonianza autentica» (WEIL, 1988, p. 37-38).
}

${ }^{7} \mathrm{Nel}$ '55 Weil scriveva a proposito: «[...] il meccanicismo dei marxisti e di certi rappresentanti delle scienze sociali e politiche accrebbe l'influenza dell'esistenzialismo e della sua considerazione della dialettica, opponendo la dialettica oggettiva alla soggettività. È stata accolta la teoria della coscienza alienata e dell'ideologia, come anche quella delle contraddizioni del sistema capitalistico della libera concorrenza [...]. Ma l'interpretazione comune del marxismo come pensiero scientifico, non filosofico, scoprendo le costanti necessarie della Storia, non il senso dei fenomeni umani e degli scopi perseguiti, respingeva verso le posizioni esistenzialiste la maggior parte dei pensatori che si interessavano di politica da un punto di vista filosofico [...]. I cristiani tra gli esistenzialisti, i personalisti del gruppo di Esprit, gli esistenzialisti atei raccolti intorno a Camus, Sartre, Merleau-Ponty (per nulla concordi tra loro su tutte le questioni) affermano e persistono nell'affermare l'importanza primordiale del soggetto. Il soggetto scopre la propria situazione: essa diviene situazione per l'uomo unicamente in questa scoperta operata dal soggetto. Nondimeno l'oggettività possiede le sue leggi, e non soltanto è legittimo, ma anche inevitabile parlare di ideologia e di analisi verace e veridica (coscienza autentica e non autentica). Ma il soggetto non è e non può essere mai sicuro di essersi liberato da ogni forma di cattiva coscienza e di malafede; se il soggetto deve sempre, almeno per poter accedere all'autenticità, assumersi e volersi come tale a partire da una decisione fondamentale, la dialettica della sua coscienza è infinita e non conosce riconciliazione. La storia ha certamente un fine, e il soggetto deve perseguirlo nella sua azione libera: è la realizzazione di una situazione nella quale ogni uomo possa assumere se stesso nella sua libertà e non sia più cosa determinata dall'esterno da parte di una società inumana (disumanizzata). Ma questo fine è perseguito nel mondo delle condizioni in cui ciascuno è condizionato, ed è impossibile decidere, se non in casi eccezionali, quando i mezzi della reificazione, cioè la violenza, non siano 
presupposti storici e filosofici, l'esegesi di Weil cercò innanzitutto di ricomprendere il legame fondamentale tra bisogno e desiderio - elementi che la lettura kojèveiana aveva pericolosamente scisso - non dimenticando di salvaguardare il valore della hegeliana dialettica oggettiva. In buona sostanza, per Weil si trattava sì di riaffermare il valore della soggettività moderna, libera e ragionevole, ma anche di non rinunciare a un monismo ontologico capace di garantire la concretezza oggettiva dei soggetti storici della trasformazione. Ed è così che Weil si rivolgeva ad Aristotele e non a Platone per sviscerare il significato di un nuovo realismo hegeliano ${ }^{8}$.

In ogni caso, l'esperienza della guerra totale e delle tirannidi moderne aveva messo definitivamente in crisi le principali categorie filosofico-politiche della modernità - "uomo" come ente necessariamente e sostanzialmente razionale, "storia" come progresso teleologicamente orientato e orientabile, "lavoro" come strumento principe dell'emancipazione umana. Tutto ciò, allora, doveva fare i conti con la questione della violenza nel mondo, con il rifiuto della Ragione e della Verità in quanto termini di un discorso solo metafisicamente assumibile 9 . Fu soprattutto in virtù di tale problema cruciale che le tesi posthegeliane di Kojève e di Weil finirono per dirigersi verso prospettive radicalmente differenti. Si trattava, in ultima analisi, di ricomprendere il significato del sapere assoluto hegeliano: se Weil ne avrebbe fornito una nuova lettura non metafisica, attraverso la rivalutazione (di certo non di stampo

richiesti e pertanto giustificati per liberare l'uomo da quelle catene di cui egli non avverte necessariamente il peso» (WEIL, 1965, p. 45-46).

${ }^{8}$ L'interesse di Weil per il pensiero aristotelico fu costante, tant'è che egli vi dedicò diversi studi nel corso del suo iter di ricerca. N'è riprova, tra le altre cose, l'interpretazione del concetto di "natura" in Aristotele, che sembrò volersi riconnettere indirettamente ad alcuni assunti fondamentali della filosofia hegeliana: «Le cose umane cambiano, invece, e non sono mai uguali a se stesse. Dobbiamo allora dire che sono riducibili agli elementi? No: ciò che qui non cambia è l'uomo, la natura dell'uomo. Ora, la natura dell'uomo, se Aristotele ha ragione, e con lui la maggior parte dei nostri contemporanei, è storica» (WEIL, 1982, p. 133-134). Non si dimentichi neppure la minuziosa analisi dei Topici, attraverso cui Weil si accinse a riscoprire la funzione determinate degli ह̌v $v \delta$ $\xi \alpha$ : le opinioni notevoli, che costituiscono centri di discorsi concreti, si differenziano dall'opinione platonica come «la semplice e vaga credenza si distingue dalle tesi tramandate, e accreditate dal consenso di tutti gli uomini qualificati, secondo la convinzione dell'uomo comune: esse hanno così la prerogativa di contenere l'esperienza dell'umanità» (WEIL, 1965, p. 65). Per una disamina efficace dell'Aristotele di Weil cfr. almeno SICHIROLLO, 1978, p. 93-114.

9 È questo uno dei principali presupposti da cui muove l'opera forse più importante di Weil: la Logique de la philosophie: cfr. WEIL, 1997. D'ora in poi indicherò quest'opera con la sigla $L F$. 


\title{
esistenzialista) di Kant e di Marx, Kojève avrebbe riconfermato la sua concezione teologico-
}

\author{
secolare della storia, che non poté che radicalizzarsi attraverso la narrazione ideologica della
}

fine "universale e omogenea", che Weil rigettò senza esitazioni10.

10 Uno dei problemi fondamentali che la $L F$ intendeva affrontare era quello della violenza nella storia, questione di
certo cruciale per tutto il dibattito filosofico francese ed europeo del secondo dopoguerra. Non sarà un caso, allora,
che nelle pagine introduttive di quest'opera si trovi un paragrafo dal titolo alquanto esplicativo: $L$ 'identità della
filosofia e della storia (cfr. $L F$, p. 98-100). Weil, infatti, rimase costantemente convinto che ogni filosofia che si
pretende sistematica non possa essere scissa dalla presa di coscienza della propria storia, dal momento che «la
filosofia si comprende solo nella sua storia e non è che tale presa di coscienza anche là dove il discorso sistematico
lo dimentica o lo nega» ( $L F$, p. 98 ). Ma quali sono gli assunti di fondo di una simile posizione? Occorre comprendere,
prima di tutto, la struttura essenziale di un'opera che, come disse Jean Wahl, rappresentò una vera e propria
«Fenomenologia dello spirito 1950» (si veda, per. es., PALMA, 2017, p. 103-5). La $L F$, così, voleva fare i conti con
l'annosa questione di quel sapere assoluto di hegeliana memoria che, per l'individuo particolare e ancora in rivolta,
non si sarebbe affatto realizzato - al contrario, per es., di quanto aveva sostenuto Kojève (cfr. $L F$, p. $473-536$ ). Tuttavia, Weil non ha mai creduto di poter mettere al bando quel carattere inevitabilmente dialettico di qualsivoglia realtà umana, oggettivamente in grado di sviluppare dei linguaggi e dei discorsi, carattere che continuava (e continua) a determinare il procedere storico segnato dalla funzione della negatività. Ed è così che in questo caso seguendo l'insegnamento di Koyré (cui pure è dedicata la $L F$ ) e poi quello di Kojève - scriveva Weil: «L'uomo non è quel che è, perché non vuole essere quello che è, non è contento di essere quello che è [...]. Egli è l'animale che parla, uno degli animali che parlano, ma è il solo animale che impiega il suo linguaggio per dire No». $L F$, pp. 15-6. Cfr. quindi KOYRÉ, 1972, p. 157-8; LFS, p. 19. Si trattava, semmai, di ricomprendere le istanze e le finalità di fondo della dialettica hegeliana: la $L F$, dunque, ci introduce all'interno di diciotto categorie filosofiche che, di fatto, hanno caratterizzato il pensiero del nostro mondo, sorte dialetticamente all'interno della violenza e della negazione del dialogo, che si sono formate in virtù di discorsi storici strutturati entro la realtà dell'uomo. Categorie filosofiche che, così, si differenziano sia dai possibili risultati di una qualsivoglia metafisica ingenua sia da ogni tentazione di presumere il discorso filosofico approdato alla sua verità finale. Le categorie che Weil va esplicitando restano centri di discorso sempre possibili, comprensione entro il discorso coerente di attitudini umane e collettive irriducibili sul piano della realtà, che nella realtà dei fatti hanno voluto e potuto inizialmente soltanto esprimersi. Sul rapporto fra attitudini e categorie cfr. $L F$, p. 100-103. In questo quadro, essenziale è il ruolo della reprise. Solo attraverso la ripresa di categorie passate, infatti, possiamo tentare di comprendere il sorgere di nuove attitudini, seppur inizialmente in modo ancora inadeguato. Sul problema della reprise e sulla natura filosofica delle categorie weiliane cfr. almeno KIRSCHER, 1992, p. 40 e 47; CAZZANIGA, 1987, p. 279-81. Qui, attraverso l'analisi della categoria weiliana de L'action, si mostra come il concetto di "ripresa" trovi le sue origini teoriche in quello (marxiano) di sussunzione formale. Tuttavia, la reprise "può anche essere sussunzione reale, adeguamento del discorso alla nuova realtà che è venuta sviluppandosi, anche recuperando in parte vecchie categorie ma in un contesto in cui la forma finale è pienamente adeguata all'oggetto (sociale e storico)» (CAZZANIGA, 1987, p. 280). Si capisce, allora, come sia solo sul terreno della storia che la filosofia possa comprendersi e comprendere: «La filosofia prima non è dunque una teoria dell'Essere, ma lo sviluppo del logos, del discorso, per se stesso e per mezzo di se stesso, nella realtà dell'esistenza umana che si comprende nelle sue realizzazioni in quanto vuole comprendersi. Non è ontologia, è logica non dell'Essere, ma del discorso umano concreto, dei discorsi che formano il discorso nella sua unità» ( $L F$, p. 100). Conseguenza che derivava da un altro fatto fondamentale: per Weil la struttura del discorso - del pensiero - non coincide mai in modo definitivo ed esaustivo con lo strutturato, con la realtà concreta. E non aver scorto tale discrepanza sul piano dell'elaborazione teorica, era stato il principale errore di Hegel e del suo sapere assoluto - un errore da cui lo stesso Hegel, tuttavia, «si libera ogniqualvolta lavora nel concreto» (Cfr. WEIL, 2003, p. 49). Ad ogni modo, il sapere assoluto hegeliano, per Weil, doveva essere kantianamente ricompreso in qualità di idea, da realizzare in un mondo ancora essenzialmente violento: si trattava di ridefinire il sapere assolutamente coerente al modo di un principio regolativo e formale da cui l'indagine filosofica deve essere guidata. La filosofia, se intende davvero fare i conti con 
In questo quadro, seppur entrambi gli autori ben seppero riconoscere le nuove tendenze culturali che s'imponevano nel dibattito francese a cavallo degli anni Sessanta, i differenti epiloghi delle loro letture hegeliane avrebbero prodotto due teorie filosofico politiche rimaste agli antipodi: se Kojève, nel bene e nel male, sembrò giustificare di fatto vecchie e nuove forme di tirannide alla luce della fine della storia e della filosofia, Weil intese salvaguardare la funzione critica del pensiero filosofico attraverso la tematizzazione di un dopo Hegel diverso da Hegel. Del resto, era stata proprio la questione della violenza nel mondo a mostrare che la ricomprensione del pensiero hegeliano - non il suo rifiuto - sarebbe stata tanto più necessaria attraverso la rilettura di Aristotele, di Marx, di Kant e del secolo dei Lumi.

\section{Una "nuova" fin de l'histoire. Il Kant di Weil tra Aristotele, Hegel e Marx}

Gli studi kantiani che Weil portò avanti nel corso degli anni Sessanta intesero quindi dimostrare una medesima tesi fondamentale: Kant aveva cercato di superare una pura filosofia dell'Essere, che era rimasta ancorata, in un modo o in un altro, ai dettami di una metafisica di stampo dogmatico e scolastico ${ }^{11}$. Si spiegano così i nuclei fondamentali dei quattro saggi su Kant raccolti in Problemi kantiani, i quali non possono che conservare un'unità teorica di fondo. Nel

\footnotetext{
la realtà, dovrà comprendere che il discorso ragionevole, universale, onnicomprensivo e sistematico può essere sempre negato con cognizione di causa: la filosofia, insomma, «resta sempre un filosofare» (WEIL, 2003, p. 49).

Non stupisce il fatto che, nella $L F$, dopo la categoria "hegeliana" dell'Assoluto, vi siano le cosiddette categorie della violenza e della rivolta - quella dell'Opera e del Finito (la categoria "esistenzialista"). Su Weil e l'esistenzialismo cfr. sicuramente BERNARDO, 2005, p. 277-90. Tutto ciò, del resto, conseguiva da una premessa cruciale, già radicalmente anti-kojèveiana: «Ogni sistema è la fine della storia, della sua storia, di quella storia senza la quale non esisterebbe e che si comprende solo in lui come storia sensata. In questo senso la filosofia è eterna perché cerca sempre la stessa cosa: la comprensione - ed è storica perché non importa quel che trova, ma per quale via lo trova, da quale punto prende inizio, in breve, qual è nella storia l'uomo che si mette alla ricerca della coerenza». $L F$, p. 119.

${ }^{11}$ Weil lo fece intendere chiaramente nella sua prefazione a Problèmes Kantiens, rimasta pressocché invariata nella seconda edizione dell'opera. Cfr. WEIL, 1980 p.13-17. Non si dimentichi che questo tema cruciale era già stato posto in nuce dall'autore nel '33, con una recensione al Kant di Gerhard Krüger, Philosophie und Moral in der Kantischen Kritik il titolo del libro, edito nel 1931. A distanza di trent'anni, del resto, il punto di partenza restava uno: come aveva ben visto Krüger, per Kant «la filosofia è una questione che coinvolge il mondo dei viventi, non una questione di gabinetto»; essa pone come suo inizio la «Lebenserfahrung, esperienza vivente e vissuta, che dà la vita e che l'informa». Cfr. KRÜGER, 1931. La recensione di Weil fu ripresa e rivista nella prefazione alla trad. fr. di M. Régnier, Critique et moral chez Kant, Beauchesne, Paris 1961; cfr. quindi «Prefazione» a G. Krüger, Critique et morale chez Kant, in WEIL, 1980, p. 185-90 (la parte qui citata si trova in p. 187). Sulla vita e sulla formazione giovanile di Weil cfr. l'utile disamina di PERINE, 1987.
} 
primo, Pensare e conoscere, la fede e la cosa in sé, Weil volle mettere in luce la differenza sostanziale tra l'attività dell'erkennen, propria dell'Intelletto, e quella del denken, propria della Ragione come tale. La differenza tra conoscere e pensare, d'altronde, era stata chiarita nella dialettica trascendentale, per come essa era stata esposta nella seconda edizione della I Critica $^{12}$. Da qui l'assunto di fondo per cui le categorie dell'Intelletto potevano determinare legittimamente un oggetto fenomenico solo se il loro uso fosse rimasto vincolato ai limiti spaziotemporali tracciati dall'estetica trascendentale. Sono queste le categorie in grado di oggettivare le intuizioni sensibili e così pervenire alla «conoscenza analitico-analizzante, in una parola: discorsiva», che è «prerogativa esclusiva dell'intelletto e delle categorie schematizzate» (cf. WEIL, 1980, p. 32). In sostanza, ogni tentativo di conoscere al di là di quei limiti spaziotemporali dati, in cui si sarebbe potuta sviluppare legittimamente un'esperienza scientifica della realtà, avrebbe indotto la Ragione a cadere nelle proprie antinomie. Come era emerso dalla stessa dialettica trascendentale, le nozioni che il pensiero dogmatico aveva preteso di conoscere al modo di semplici oggetti, l'“Anima", il "Mondo" e "Dio", rimanevano altresì concetti vuoti, senza intuizione. Tanto più che «solo la percezione (l'intuizione) può rispondere alla domanda: che cos'è? mentre il concetto indica solo la forma dell'unità, unità che unifica il sensibile» ( WEIL, 1980, p. 33). In poche parole, i concetti "vuoti" della ragione (Anima, Mondo, Dio) potevano essere solo pensati come principi regolativi dell'esperienza in quanto tale, idee che avrebbero permesso all'individuo concreto di scorgere la possibilità di un senso ragionevole e universale del proprio discorrere e del proprio agire ${ }^{13}$. Weil, del resto, lo aveva scritto nella

\footnotetext{
${ }^{12}$ Sulle differenze tra I e II edizione della Critica della ragion pura cfr. WEIL, 1980, p. 19-23.

${ }_{13}$ Sul sistema delle idee cosmologiche si veda quindi KANT, 1967, p. 354-361; sulle antinomie della ragione cfr. KANT, 1967, p. 366-395. Per quel che riguarda il problema della reificazione delle idee, ad opera del dogmatismo scolastico, si veda quanto sostenuto a buon diritto da Pasquale Salvucci interprete di Weil: «Lo scopo al quale Kant ha mirato è, per il Weil, la "metafisica positiva". La Prefazione del 1787 (della I Critica) si presenta duramente polemica contro il modo di vedere di molti suoi lettori che, considerando soltanto "l'aspetto negativo della Critica", hanno ignorato la "funzione positiva" della "limitazione della ragione costruttivistica", attuata dalla Critica, limitazione che ha reso la ragione, che precedentemente era presuntuosa, cosciente dei propri limiti e in grado, per ciò stesso, di accedere alla "metafisica positiva" ben diversa dalla illegittima metafisica dommatico/costruttivista che pretendeva, che pretende di realizzare un uso trascendentale (che la Critica ha mostrato illegittimo, un "non-uso") delle categorie nel conoscere, come accade nelle Scuole filosofiche» (SALVUCCI, 1989, p. 100). Sui mutamenti di significato del termine "trascendentale" dal medioevo alla modernità si veda almeno BARALE, 2007, p. 53-72.
} 
prefazione del ‘63 a Problemi kantiani: «[...] la Critica della ragion pura parla della scienza solo per fondare una morale» (WEIL, 1980, p. 15). Esisteva, dunque, una sola e unica Ragione: la ragione in quanto pratica, il cui interesse fondamentale era quello di preservare la ragionevolezza e la sensatezza della convivenza concreta fra gli uomini.

Fu questo il punto essenziale che Weil volle sviluppare nel suo secondo saggio su Kant, Senso e fatto. In quest'ultimo, indagando i problemi di fondo della III Critica kantiana, l'autore arrivò a sostenere una tesi di certo innovativa per gli studi dell'epoca. Per Weil infatti, con la scoperta del giudizio riflettente, Kant era riuscito a mostrare in che misura l'individuo ragionevole avrebbe potuto ritrovare, nella realtà particolare, il senso dei fatti dapprima scientificamente determinati ${ }^{14}$. Le condizioni di possibilità dell'unità del mondo, dello scopo universale della Storia e della libertà dell'agire morale, dovevano mostrarsi nella contingenza in cui vivevano gli individui concreti. Ecco perché la III Critica volle parlare di una teleologia della natura che, pur non trovando riscontro nelle leggi oggettive della scienza, poteva in ogni caso essere rintracciata da chi se ne fosse messo in cerca. Interpretare i fenomeni naturali come se essi fossero davvero parte di un sistema teleologico e unitario, infatti, avrebbe permesso agli individui di pensare l'idea della Storia universale in quanto incedere progressivo dell'umanità verso la realizzazione della società del genere umano ${ }^{15}$. Tuttavia, di tale finalità ultima non si poteva affatto dimostrare o dedurre la necessità assoluta, come avrebbe preteso una metafisica

\footnotetext{
${ }^{14}$ Scrive Weil: «L'attività giudicatrice assume così un nuovo compito. Fin qui la sua sola funzione era stata quella di sussumere il dato sotto i concetti, sia fondamentali sia costruiti a partire dall'esperienza; il nuovo problema fa che essa cambi direzione nel suo lavoro e, invece di classificare il dato sensibile sotto il dato teorico, si metta a cercare il concetto sotto il quale poter collocare quello che essa incontra. Essa cessa di essere determinante e si fa riflettente» (WEIL, 1980, p. 70). Il tutto si può spiegare così: se nella I Critica si era posto quel livello d'esperienza possibile che delimita una dimensione sintattica di ogni nostro legittimo e logico determinare, nella III Critica si scopre una dimensione riflessiva del giudizio che deve esser portata a consapevolezza. L'individuo che si pensa come essere intenzionale deve ritrovare gli effetti del suo agire nel mondo, deve ritrovare il senso di quel discorso svolto dall'esperienza scientifica del conoscere: deve ritrovare quella dimensione semantica che possa ostensivamente restituire il valore di ogni oggetto di un mondo di cui questa Ragione, essenzialmente discorsiva, si è fatta unico soggetto possibile. La distinzione tra "dimensione sintattica" e "dimensione semantica" di cui qui è questione è stata ben evidenziata da Massimo Barale (1988, p. 22). Si veda quindi KANT, 1995. Si consideri che Weil tradusse il titolo tedesco «Kritik der Urteilskraft» con «Critique de la Judiciaire», volendo così sottolineare il carattere (pro)attivo (e "giurisdizionale") del giudizio che riflette e si riflette.

15 Cfr. KANT 1995, p. 241-45. Di fondamentale importanza, quindi, erano le capacità simboliche e analogiche del pensiero discorsivo e ragionevole. In merito si veda SALVUCCI, 1989, p. 112-117.
} 
dogmatica: se la «teleologia naturale s'arresta così sulla soglia, ma dopo averci condotto fino ad essa» ciò era dipeso dal fatto che questa «resta incapace di scoprirci il senso finale» (WEIL, 1980, p. 90). Resta il fatto che è sempre tale teleologia che «legittima il presupposto, inevitabile per il pensiero finito dell'uomo, di una finalità della natura e una interpretazione di ciò che è secondo il tipo di ciò che è stato voluto da una intelligenza» (WEIL, 1980, p. 90). Ed è così che «essa ci conduce - questo merito è grande - all'idea di un essere superiore» (WEIL, 1980, cit., p. 90). Si rivelava in tal modo il carattere prettamente regolativo e formale dell'idea di Dio, capace di garantire un senso ultimo e unitario della Storia solo per colui che si fosse deciso a ricercarlo ${ }^{16}$.

In breve, i fatti conosciuti attraverso la spiegazione e l'analisi dell'Intelletto avrebbero acquisito un senso sistematico e unitario per l'uomo soltanto ammettendo che la natura poteva essere pensata «come strutturata e sensata» (WEIL, 1980, p. 94). È questa medesima idea, pertanto, che avrebbe indotto l'individuo agente a pensarsi come essere finito e, allo stesso tempo, libero. Libero nei limiti di un mondo in cui egli avrebbe potuto e dovuto ritrovare gli effetti concreti della sua azione ragionevole, giacché proprio «una natura sensata gli permette questa scoperta e ve lo sospinge» (WEIL, 1980, p. 94) ${ }^{17}$. Tutto ciò, lo ripetiamo, non avrebbe potuto più fondarsi su alcuna necessità metafisica di sorta, incapace di riconoscere il valore della soggettività in quanto infinita negatività agente: «L'esistenza degli esseri morali e ragionevoli, non la soddisfazione dell'appetito naturale di questi esseri - ecco chi fonda ora tutto. Tutto, perché anche la teleologia naturale è in ultima analisi il prodotto di quella facoltà dei fini che caratterizza l'essere morale» (WEIL, 1980, p. 91). Insomma, l'auto-riflessività del giudizio porta l'uomo a comprendere che «per lui si tratta di orientarsi, e nei due sensi di questa

\footnotetext{
${ }^{16}$ Dio, in sintesi, poteva esistere solo per l'uomo ragionevole: «Dio [...] non si mostra all'uomo osservatore, all'uomo di scienza. Dio come idea è solo un principio di "comprensione", non di spiegazione, di scienza. Si tratta di comprendere che la stessa scienza concreta diventerebbe inconcepibile in un mondo non-organizzato e non orientato; si tratta di comprendere che la realtà naturale e storica è un intero "sensato" ma per l'uomo, sensato (solo) per mezzo dell'uomo e che appare come "aggregato insensato" all'uomo che non si eleva al senso e alla "comprensione del senso del mondo e del senso nel mondo"» (SALVUCCI, 1989, p. 110).

${ }^{17}$ In questo quadro, di fondamentale importanza restava la nozione di "causa intellegibile" che Kant sviluppò già nella I Critica: cfr. KANT, 1967, p. 443-444.
} 
parola: trovare il polo e le coordinate naturali della propria esistenza, e porsi nella giusta direzione» (WEIL, 1980, p. 95).

Arriviamo così a un punto cruciale, perché è qui che emerge una delle differenze sostanziali che, per Weil, intercorrerebbe tra il pensiero platonico e quello kantiano. Seppur l'istanza di orientamento e di orientabilità dell'agire umano concreto, infatti, «si applicherebbe, per esempio, anche alla filosofia platonica, che promette un orientamento simile» (WEIL, 1980, p. 95), tuttavia

la differenza tra le due resta nondimeno considerevole, addirittura di essenza. Per Platone il sapere e la visione ( $\theta \varepsilon \omega \rho i ́ \alpha)$ della struttura sensata, dell'Idea del Bene esistente (sovra-esistente), guidano l'uomo; per Kant polo, direzione e determinazione, si trovano tutti e tre non nella natura, nel cosmo, ma nell'uomo come essere agente. Sono io che colgo nella conversione alla libertà ragionevole, nella comprensione immediata del mio fondamento la possibilità di dare un senso alla mia esistenza e quindi a quella del mondo. Per l'uomo puramente teoretico il mondo non è che un vano spettacolo, il gioco insensato di un creatore semplicemente abile, e in realtà rimarrebbe tale se non vi introducessi la questione del valore (termine kantiano) e del senso (termine che preferiremmo, considerate la sociologizzazione e la storicizzazione del primo, entrambe dovute al pensiero postkantiano), se io non realizzassi nei miei atti quella libertà che solo è valore da (e per) essa stessa e che, in quanto ragione, pone la questione del senso. Il mondo possiede senso e valore nella misura e solo nella misura in cui l'uomo si pone nella sua azione e per sua scelta come senso rendendo sensata la propria vita (WEIL, 1980, p. 95) 18 .

Fatta salva l'evidente presa di distanza dalle tendenze "sociologiche" e "storiciste" del suo tempo, pare altrettanto evidente come qui Weil intendesse scongiurare una riduzione della filosofia kantiana a dettami teorici puramente dualistici e soggettivistici ${ }^{19}$. L'uomo può e deve (soll) ritrovare il Cosmo, dacché il fine in sé della Storia - in sé: cioè non conoscibile nella sua configurazione empirica definitiva - resta, secondo l'interesse e il fatto della Ragione, «la realizzazione di un mondo armonioso secondo quel fine della libertà che è la libertà stessa»

\footnotetext{
${ }^{18}$ Non a caso, Weil ci dice che Kant «ha fatto di tutto per attenuare la sua prova» ontologica dell'esistenza di Dio, dal momento che «la sua forza, se si fosse potuta giudicarla irresistibile secondo la metafisica cosificante, sarebbe stata la sua disfatta. La questione del senso non si pone, non può porsi che per l'essere-in-sé nel mondo, per la volontà ragionevole determinantesi essa stessa nella condizione, non per la ragione teorica che in quanto tale è solo riflessione sull'intelletto e sui suoi metodi di acquisizione di conoscenze (sebbene questa ragione, se comincia a riflettere su se stessa, finisca per scoprire l'inanità dell'intelletto e si scopra come interessata, pratica)». Cfr. WEIL, 1980, p. 98.

${ }^{19}$ Sui quali, al contrario, si era fondata tutta l'interpretazione hegeliana di Kojève.
} 
(WEIL, 1980, p. 97) ${ }^{20}$. Si capisce allora perché l'essere finito che trasforma e domina la natura (o la "seconda natura") non possa oltrepassare «ogni dato se non in quanto membro del mondo [...] essere finito che supera la condizione, perché la condizione lo prepara a questo superamento. Natura e libertà sono indissolubilmente legate» (WEIL, 1980, p. 97) ${ }^{21}$. Ne conseguiva che il sistema kantiano, per Weil, sarebbe riuscito a porre «il problema del senso che è, del senso esistente» (WEIL, 1980, p. 106), senza per questo sprofondare del tutto negli abissi della filosofia dell'Essere. Tanto più che se Kant, da un lato, «scopre che ogni essere delle cose non si comprende che a partire da un senso esistente, reale, anteriore a ogni fatto e a ogni dato», dall'altro egli riconosce che «fatto e dato non si rivelano se non a colui che li interroga nella sua propria ricerca del senso» (WEIL, 1980, p. 106-107). Resta pur sempre il fatto che

Kant non osa passare esplicitamente da una filosofia dell'essere (nella quale ricadrà Hegel, dopo l'insuccesso del grande tentativo fichtiano, che voleva dedurre e costruire la realtà a partire dal senso) a una filosofia del senso. 0 piuttosto, poiché la formula non è sufficientemente precisa, non osa parlare un linguaggio che possa esprimere l'unità indissolubile di fatto e senso: ogni fatto è sensato, ogni senso è (WEIL, 1980, p. 107).

Ciò che qui occorre mettere in luce, allora, sono due aspetti essenziali: per il Weil degli anni Sessanta, se Kant era riuscito a porre, seppur implicitamente, un'autentica filosofia del

\footnotetext{
${ }^{20}$ In quest'ottica, «sono i corpi viventi e le cose belle o grandiose che forniscono all'uomo il simbolo della cosa in sé: all'uomo che, ponendo la questione del senso dell'esistenza del mondo e della propria, diventa la coscienza nella quale il mondo si sa mondo ed è compreso come unità naturale per una volontà non-naturale, ma incarnata nel mondo. Questa ragione-volontà è simbolizzata nel mondo. Il fatto diviene comprensibile solo a partire dalla finalità, in funzione della finalità, ogni comprensione (in ciò essenzialmente differente da ogni conoscenza) parte dall'idea di un fine oggetto di mira ed è completa solo al momento in cui il fine si rivela come interno, fine in sé, rapportato a sé e soltanto a sé» (WEIL, 1980, p. 96). Lungi dall'essere un mero interprete dell'esser dato, l'uomo, in Kant, «non è quindi spectator mundi» (WEIL, 1980, p. 100).

${ }^{21}$ Sulla categoria della condizione, in grado di restituire i tratti costitutivi della società del lavoro ("seconda natura" o "pseudonatura" dell'uomo moderno) cfr. ancora una volta la $L F$, p. 283-322. È certo, comunque, che siamo ormai di fronte a un kantismo quantomai distante dal dualismo soggettivistico di matrice kojèveiana. Piuttosto, come ha notato molto acutamente Patrice Canivez, il Kant di Weil sembra in qualche modo ritornare ad un Aristotele ricompreso dopo Hegel (nel senso indicato dalla $L F$ ): «[...] la realtà per Kant si costituisce a partire dal contingente. Il reale è più del possibile, seppur non sia necessario [...]. Questa realtà è, allora, fortuita. A partire da qui, la realtà del senso è essa stessa contingente. È per questo che si ha bisogno di un Essere supremo per garantire il senso del mondo, per garantire la realtà del senso. Di contro, la natura come un cosmo è, allo stesso tempo, una sorta di aristotelismo. Se volessimo giocare con le definizioni, potremmo dire che la filosofia di Eric Weil non è soltanto un kantismo post-hegeliano, ma anche un aristotelismo post-kantiano - come anche un hegelismo post-marxista» (CANIVEZ, 2014, p. 25-6).
} 
senso, Hegel, al contrario, era ricaduto precisamente in una metafisica del senso o filosofia dell'Essere, finendo per affermare la deducibilità e la necessità onto-logica del Senso ${ }^{22}$. Torniamo quindi a Problemi kantiani. Negli ultimi due saggi qui raccolti, Weil non fece altro che analizzare le ricadute pratiche del pensiero di Kant così interpretato. Il punto centrale della questione, allora, tornava a essere quello dell'orientabilità del progresso storico e umano, in vista della realizzazione del regno dei fini sia attraverso l'azione politica ragionevole sia in virtù della moralizzazione del genere umano ${ }^{23}$. Come ha ben sintetizzato sempre Salvucci:

La stessa natura diventa «comprensibile» solo con il soccorso dell'idea di finalità. E così la storia. La stessa natura vuole (per chi si accosta ad essa, con l'idea di finalità) che gli uomini, una volta usciti dall'infanzia della specie, perseguano con le loro azioni libere il fine supremo, quello «stato cosmopolitico universale che dovrà essere il seno materno nel quale si svilupperanno tutte le

${ }^{22}$ Tesi già posta da Weil, come visto, nella $L F$ (cfr. supra nota 10). È vero anche, però, che «la lettura di Kant fatta da Weil, specialmente in Sens et Fait, praticamente coincide con la lettura che ne aveva fatto Hegel. Il quale polemizza con Kant, ma riconosce (e Weil altrove sottolinea questo punto) che Kant era andato oltre la riflessione, ma non aveva tratto tutte le conseguenze perché prigioniero della sua idea del conoscere soggettivo finito [...]» (VALENTINI, 1989, p. 144, nota 9, i corsivi sono miei). A riprova di questa considerazione di Valentini (come lui stesso ha ricordato) vi è soprattutto il § 55 dell'Enciclopedia hegeliana, che recita: «La Critica del giudizio ha questo di notevole, che Kant ha con essa espresso la rappresentazione, e anzi il pensiero, dell'Idea. La rappresentazione di un intelletto intuitivo, di una finalità interna ecc. è insieme l'universale pensato come concreto in sé stesso. Perciò soltanto in queste rappresentazioni la critica kantiana si mostra speculativa. Molti, e specialmente Schiller, hanno trovata nell'idea del bello d'arte, dell'unità concreta del pensiero e della rappresentazione sensibile, la via d'uscita dalle astrazioni dell'intelletto [...]. Così il prodotto d'arte come l'individualità vivente sono, a dir vero, limitati nel loro contenuto: ma l'idea, comprensiva anche secondo il contenuto, è concepita da Kant e riposta nella postulata armonia della natura o necessità col fine della libertà, nello scopo finale del mondo che si pensi come realizzato [...]. Invece, la presenza delle organizzazioni viventi e del bello d'arte è bastevole a mostrare, anche al senso e all'intuizione, la realtà effettiva dell'ideale. Le riflessioni kantiane su questi oggetti sarebbero dunque particolarmente adatte a introdurre la coscienza nella comprensione e pensamento dell'idea concreta» (HEGEL, 2009, p. 68-9). Qui Hegel, evidentemente, volle rimandare anche al kantismo di F. Schiller (2009).

${ }^{23} \mathrm{Si}$ veda in particolare Il male radicale, la religione e la morale, in cui Weil analizza la concezione antropologica kantiana che definisce l'uomo come "legno storto", un essere che oscilla costantemente tra passione e ragione, tra violenza e discorso. E che dunque andrà educato alla ragionevolezza. Cfr. WEIL, 1980, p. 149-81. Una visione, quella kantiana, che anche in questo caso era stata effettivamente influenzata dal pensiero aristotelico. Nell'Etica Nicomachea, infatti, leggiamo: «Si deve indagare quali sono le cose verso cui noi siamo inclini, dato che persone diverse sono portate per natura a cose diverse. Questo risulterà chiaro dal piacere e dal dolore che nascono in noi. Dobbiamo spingerci nella direzione opposta, infatti allontanandoci molto dall'errore perverremo al giusto mezzo, come fanno coloro che raddrizzano i legni storti» (ARISTOTELE, 2009, p. 75). Cfr. quindi la VI tesi di KANT, 1995, p. 35-6. Si giustificano così le conclusioni di Pasquale Salvucci sul problema dell'orientabilità del movimento storico universale: «Ma pensare la storia come se essa fosse orientata da un filo conduttore, come avente direzione e senso per l'uomo che così la pensa, non si risolve, per Kant, in una previsione infallibile, perché, come egli stesso ammonisce, è sempre possibile che l'uomo, ente libero, con barbarica devastazione, distrugga di getto tutta la stessa civiltà faticosamente conquistata sin qui [...]» (SALVUCCI, 1989, p. 112). Non era forse questo il problema di fondo che avevano (ri)compreso le categorie posthegeliane della violenza nella $L F$ ? 
originarie disposizioni della specie umana». Lo scopo finale assoluto del mondo sta nella realizzazione della libertà ragionevole di tutti e di ciascuno... (SALVUCCI, 1989, p. 110) ${ }^{24}$.

Ecco svelato il vero legame con il Marx dei Manoscritti economico-filosofici o con quello del III libro del Capitale, lì dove in gioco vi era un'altra nozione fondamentale capace di illuminare la ragion d'essere di questo "kantismo marxiano": quella di necessitò condizionale 25 . In tal senso, gli individui della condizione ("pseudonatura" o "seconda natura") sarebbero pervenuti alla loro vera umanizzazione solo se la loro azione fosse stata effettivamente orientata all'emancipazione materiale e morale di tutti e di ciascuno ${ }^{26}$. Arriviamo così a un punto fondamentale, dal momento che tutto questo complesso teorico finiva per ricomprendere

${ }^{24} \mathrm{Si}$ comprende perché l'uomo possa esser definito, kantianamente (e aristotelicamente), un animale ragionevole, che può farsi ragionevole e pervenire, così, alla realizzazione del regno dei Fini e della Libertà. Si veda in particolare l'VIII tesi di Kant in Idea per una storia universale dal punto di vista cosmopolitico.

${ }^{25} \mathrm{Ne}$ ha dato conto, alquanto weilianamente, André Tosel. Che, così, ha di fatto offerto una nuova spiegazione della filosofia della storia marxiana: «La configurazione che unisce processualità critica della produzione e sistema [...] non è fondata su una necessità metafisica - seppur Marx, per preservare l'obiettività della teoria della storia e la sua ineliminabile naturalità, la rifletta in modo discutibile nelle formule ispirate dalla filosofia speculativa hegeliana. È vero che Marx sostiene di aver per oggetto le "leggi naturali della produzione capitalistica [...] queste tendenze che agiscono e si fanno valere con ferrea necessità"; [che] "il mio punto di vista [...] considera lo sviluppo della formazione economica della società come processo di storia naturale". 0 ancora: "una società [...] non può né saltare né togliere di mezzo con decreti le fasi naturali dello svolgimento" [cfr. MARX, 1996, p. 42-43]. È vero anche che Marx rivendica la dialettica [che] "nella comprensione positiva della realtà delle cose include nello stesso tempo anche la comprensione della negazione di essa e del suo inesorabile declino" [cfr. MARX, 1996, p. 49]. Tali affermazioni [...] impediscono di cogliere che la determinazione [della necessità] che Marx considera è condizionale. L'evidente difficoltà sta nel fatto che questa necessità condizionale (del tipo se...allora) opera nel senso d'un condizionamento altamente direttivo o, piuttosto, unidirezionale: il modo di produzione della vita materiale condiziona in generale il processo della vita sociale, politica, culturale e i sistemi d'interazione normativa. Non si tratta di fatalismo metafisico, ma della determinazione di ciò che costituisce il movimento oggettivo peculiare ai diversi "contesti artificiali" e umani, distaccatesi nel tempo dalle condizioni naturali originarie. La sequenza dei modi di produzione è una logica che permette la determinazione di un processo storico in cui ciascuna fase non contiene in sé la successiva. E questa logica ha un contenuto che può essere "mostrato": essa non si costituisce a partire da un presupposto metafisico, giacché indica l'effettiva capacità sempre maggiore di dominare il ciclo naturale, la tendenziale emancipazione del lavoro necessario e quindi dell'ordine vitale, fatti salvi i problemi posti da tale "progresso". Si tratta di una determinazione oggettiva condizionale, tendenzialmente unidirezionale, ma nient'affatto di un determinismo necessitarista» (TOSEL, 1987, p. 141, gli ultimi due corsivi sono miei).

${ }^{26}$ Assunti che erano già stati posti in altri termini nella $L F$, più precisamente nella categoria weiliano-marxiana de L'azione - che si determina dialetticamente (nel senso indicato da Weil) dopo quella del Finito. L'azione era stata introdotta come segue: «La finitezza, rivelando la condizione umana, si rivela semplice discorso all'uomo che si oppone alla violenza sul piano della violenza: quest'uomo agisce sulla realtà nella sua totalità per sottometterla al suo discorso». $L F$, p. 537. 
criticamente - e in senso non ideologico - proprio la teoria della fin de l'histoire inaugurata, più di vent'anni prima, da Kojève.

In tal senso, la prospettiva posthegeliana di Weil che, di fatto, intese ricomprendere istanze kantiane e marxiane insieme, sarebbe emersa definitivamente in un saggio del '70, dedicato proprio a La fin de l'histoire. Titolo che non lasciava spazio ad alcun dubbio sull'oggetto della ricerca: si trattava di far chiarezza una volta per tutte su cosa fosse davvero la "fine della storia". Del resto, fu proprio con questo studio che si consumò definitivamente la rottura con le tesi kojèveiane. Weil lo fece intendere sin da subito: «La fine della storia: l'espressione è di moda, o almeno lo è stata per molto tempo. Forse, è soprattutto il marxismo a portarne la responsabilità o il merito $\mathrm{o}$, forse, una certa interpretazione della filosofia hegeliana - pur sempre di dubbio valore» ${ }^{27}$. Restava il fatto che il sogno della fine della storia, come compiuta realizzazione del migliore dei mondi possibili, sembrava progressivamente svanire di fronte a un mondo che andava verso tutt'altra direzione. Cionondimeno le tesi sostenute dai difensori della fin de l'histoire erano note: «Noi viviamo ancora nella storia, ma è sempre più vicino il giorno in cui ne usciremo, in cui ne saremo usciti; di più: de iure la storia è già terminata e se essa, nei fatti, sembra durare ancora è solo a causa della nostra inconsapevolezza [...]» (WEIL, 2003, p. 167). In sintesi, soltanto per via di questa inconsapevole insipienza dei più verremmo ancora a conoscenza di «guerre, di rivoluzioni, di ingiustizie e di lotte di liberazione» (WEIL, 2003, p. 167). Il riferimento sottaciuto alla lettura kojèveiana della fine - che si radicalizzò in senso ancor più dogmatico sul finire degli anni ‘60 - mi pare evidente ${ }^{28}$. Non a caso, Weil non

27 Cfr. WEIL, 2003, p. 167-76, in particolare p. 167.

${ }^{28}$ Massimo Palma si è interrogato sulle ragioni storiche che avrebbero indotto Weil a tornare, solo nel '70, su un tema del genere: «È il 1970 ormai. Alexandre Koyré e George Bataille, icone di apertura e di chiusura del cerchio mitologico della fine della storia, sono ormai morti da anni. Con loro, da poco, è scomparso anche Alexandre Kojève, che del mito fu banditore volontario. Che senso ha scriverne ancora, quando le aspettative destate dal secondo conflitto mondiale sono scemate nelle aporie della Guerra fredda da un lato, nelle turbolenze delle nuove generazioni dall'altro? [...]». È chiaro che Weil volle in qualche modo rispondere all'aggiunta sulla "fine della storia" che Kojève aveva apportato nella seconda edizione dell'LFS, uscita solo due anni prima del saggio weiliano in oggetto. Palma ha aggiunto a riguardo: «Da decenni, ormai, quell'idioma è comune a quella generazione invecchiata e in parte svanita, ma la Nota alla nota ha vita giovane». Cfr. PALMA, 2017, p. 127. Del resto il tema della fin de l'histoire, pur avendo perso la sua forza ideologica o mitologica nel corso degli anni, è rimasto per lungo tempo (se non ancora oggi) uno dei "mantra" del pensiero neoliberale, non fosse che per merito o per colpa di Francis Fukuyama e della cosiddetta scuola di Chicago (a riguardo cfr. sicuramente F. FUKUYAMA, 2017). Basti pensare che lo stesso Fukuyama è tornato sulla questione più volte nel corso degli ultimi anni. Ironia della sorte (forse a 
poté che problematizzare in toto le tesi in oggetto, intendendo difendere ancora una volta la capacità critica della Vernunft, alla luce dell'effettualità del movimento storico-dialettico. Ché le guerre, le rivoluzioni e le lotte di liberazione ancora in atto non si sarebbero potute più giustificare di fronte «al giudizio della ragione», qualora esse fossero state considerate «necessarie» soltanto a «svelare, a noi ottusi, ciò che il pensiero di uomini illuminati [clairvoyants] ha già riconosciuto da molto tempo: la fine inevitabile e a un tempo felice della storia» (cf. WEIL, 2003, p. 167) ${ }^{29}$.

Ebbene, una volta constatato che l'operare effettivo della negatività storica non si era affatto esaurito, Weil intese smitizzare il sogno della fin de l'histoire ribadendo che quest'ultima né si era già realizzata né tantomeno poteva più fondarsi su presunte teologie della storia ${ }^{30}$. L'autore lo riaffermò in modo chiaro: «L'umanità non desidera affatto la propria scomparsa.

causa della crisi economica, sociale, politica e culturale che ormai perdura in Occidente da almeno un decennio, in tutta la sua violenza) Fukuyama, in un'intervista concessa al magazine britannico New Statesman uscita il 17 ottobre 2018, ha ammesso che il «socialismo dovrebbe tornare». Cfr. l'intervista integrale di George Eaton:

https://www.newstatesman.com/culture/observations/2018/10/francis-fukuyama-interview-socialism-oughtcome-back.

${ }^{29}$ È anche per questo che Weil volle ripensare in chiave realistica, non escatologica né tantomeno teologica, la "fine della storia". Era evidente, nei fatti, che non si sarebbe potuto più parlare di fin de l'histoire come evento finale (dalle caratteristiche apocalittiche) in cui «l'umanità scompare» e «non c'è più storia». L'autore non lascia spazio a dubbi: «È chiaro che non è a questa fine della storia che pensiamo quando se ne promette la fine. Non immaginiamo affatto la liquidazione radicale del tempo, né la fine degli avvenimenti, né la morte universale. Al contrario, si parla della fine della storia come di un evento auspicabile, dalle conseguenze piacevoli e felici. Supponiamo, dunque, che una volta passato il momento decisivo, gli esseri umani saranno ancora là per fruire di quel che noi, forse, potremmo chiamare post-storia» (WEIL, 2003, p. 168).

${ }^{30}$ In tal senso, sarà utile ricordare quanto ricostruito da Marco Filoni sulla formazione e sugli sviluppi del pensiero di Kojève: «Giunto a Parigi nel '26, dopo gli studi in Germania, iniziò a frequentare gli intellettuali e i teologi dell'emigrazione russa, convinti del ruolo messianico del loro paese natale: il filosofo Lev Karsavin, il teologo Georgij Florovskij e Nikolaj Berdjaev. E anche negli anni successivi preferì discutere con importanti figure del pensiero cattolico, in prevalenza gesuiti, come Gaston Fessard, Henri Bouillard ed Edmond Ortigues. A partire dai primi anni Cinquanta, e per oltre quindici anni, questi tre filosofi e teologi presero l'abitudine di incontrarsi a casa di Kojève almeno una volta al mese, per cenare e poi discutere fino a tarda notte. Fu proprio in una di queste serate che Kojève confidò agli amici il motivo per cui persone come Sartre, Eric Weil o Maurice Merleau-Ponty non comprendevano affatto il suo pensiero: "perché non sono teologi!". Egli era pienamente consapevole dell'esistenza di un passaggio dalla teologia alla saggezza non-teologica, ovvero di quella che lui chiamerà "fine della storia". Ma la fine della storia altro non era per Kojève che la fine della teologia. Egli pensava che l'uomo in quanto tale esistesse da millenni solamente in quanto essere che ha un Dio o degli dèi. E la fine della storia era esattamente il momento in cui Dio scende sulla Terra e diventa umanità o, se si preferisce, spirito. Per Kojève era giunto quel momento, e si trattava ormai di pensare il Dio ateo, giunto sulla Terra» (FILONI, 2013, p. 24). Non bisogna neppure dimenticare che Kojève, non a caso, aveva dedicato la sua tesi di dottorato (sotto la guida di Karl Jaspers) al pensiero del teologo Vladimir Sergeevič Solov'ëv, il cosiddetto "Hegel russo". Cfr. in merito TAUBES, 1996, p. 76; quindi KOJÈVE, 2009, p. 21-92. 
Essa, almeno nella nostra tradizione mediterranea, greca e giudaico-cristiana, non sogna neppure la totale entropia o il nirvana: essa vuole vivere e prender parte a ciò che accade, vivere il tempo, il suo tempo e farlo felicemente» (WEIL, 2003, p. 169). Era piuttosto l'idea di finalità, mitizzata dall'ideologia della fine, che doveva assumere di nuovo una funzione essenzialmente regolativa e un carattere del tutto formale: ciò che si poteva e si doveva continuare ad avere in vista, sotto la guida della Ragione, era la fine di una storia di ingiustizie e di disumanizzazioni, da attuare attraverso una prassi storica che occorreva rielaborare costantemente di fronte alle necessità reali del proprio presente ${ }^{31}$. Di cruciale importanza, pertanto, si confermava il valore della soggettività moderna in quanto negatività agente, capace di trasformare la realtà all'interno e in virtù di condizioni (pseudo)naturali date, comprendendosi così come potenziale artefice del proprio destino. Quest'ultimo, in breve, non avrebbe più dovuto mostrarsi come una cieca fatalità 32 .

Non sarebbe difficile intravedere in tali posizioni weiliane una sorta di afflato nostalgico per un mondo moderno figlio del secolo dei Lumi, come d'altronde è stato notato ${ }^{33}$. È un fatto che questo sguardo sistematico sul mondo, questo non voler rinunciare alla possibilità dell'emancipazione umana, svelava istanze critiche che stavano perdendo la loro forza di fronte agli sviluppi più recenti delle società occidentali. Weil, però, ne fu perfettamente consapevole: egli tentò di contrastare coscientemente - con scarsi risultati, non c'è dubbio - tendenze politiche e speculative che, passando per la legittimazione di vecchie e di nuove tirannie, avevano iniziato ad imporre un modo postmoderno e post-dialettico (sul piano politico: post-

\footnotetext{
31 «Ciò che allora vogliamo veder arrivare alla sua fine è, evidentemente, non la storia, ma la storia cattiva [mauvaise], la storia come male. In fondo, e questo fondo si mostra ora, noi non vogliamo la fine della storia, ma la fine del male e dei mali - la fine non già della storia, ma di una storia che è malvagia perché storia di un'epoca dominata dal male e dalla sofferenza» (WEIL, 2003, p. 169). Alla luce di tutto ciò, Massimo Palma ha potuto affermare che, dopo Kojève e con Weil, «la fine della storia da escatologia diventa teleologia» (PALMA, 2017, p. 127). Se da un lato è indubbiamente corretto desumere una simile conclusione, è altrettanto importante non dimenticare che tale finalismo storico, in Weil, non è affatto necessario che sia (né può permettere la predeterminazione positiva dell'avvenire). Tornerò a breve su questo punto.

${ }^{32}$ Con Kant (e con Weil), potremmo dire che il destino dell'uomo morale doveva farsi Bestimmung e non rimanere soltanto insensato Schicksal.

${ }^{33}$ Cfr. soprattutto ROTH, 1988, p. 176-83, in cui uno dei paragrafi dedicati a Weil s'intitola «Between Hope and Nostalgia».
} 
democratico) di concepire la realtà (post)umana e (post)storica. Ma per Weil era proprio per queste ragioni che occorreva uscire dal regno delle necessità e dei bisogni con un'urgenza ancora maggiore. Era ancora il problema dell'uomo, infatti, a mostrare pur sempre che «noi neghiamo ciò che vogliamo che scompaia e siamo convinti che la vita vera, la vita veramente umana, contenta e felice, comincerà e durerà a partire da questa fine della storia» mauvaise (cf. WEIL, 2003, p. 172). L'uomo concreto, non bisogna dimenticarlo, sa soltanto ciò che non vuole, che la sua vita e il suo mondo «non sono quel che dovrebbero essere» (WEIL, 2003, p. 171). Egli può scoprire ciò che lo rende davvero felice soltanto via negationis: la felicità finale non ancora raggiunta sarà «assenza del male, assenza di costrizione» (WEIL, 2003, p. 172).

Ecco che riemerge tutta l'importanza pratica del formalismo della Morale: si trattava ancora di portare a compimento la libera emancipazione degli individui dalla violenza e dal loro stato di insoddisfazione. Di assicurare, cioè, il pieno sviluppo delle facoltà umane e ragionevoli così da pervenire al riconoscimento universale di tutti e di ciascuno nella pluralità. Del resto, positivizzare l'Idea di ciò che non potrebbe non renderci contenti, veramente soddisfatti e felici, avrebbe significato ricadere nell'omogeneità totalizzante della fine della storia kojèveiana. Ecco perché della fin de l'histoire non si poteva che parlare

in termini negativi, se dobbiamo rimanere liberi e se solo noi stessi, nessun altro - soprattutto gli esseri non liberi quali siamo ancora oggi - non può né deve voler determinare in anticipo il contenuto di questa vita a venire, che, se potesse esser determinata in anticipo, non sarebbe affatto libera: non si può, io non posso impormi il contenuto della mia felicità futura. Devo rispettare la mia libertà a venire, la mia speranza di felicità, la realizzazione di me stesso ad opera di me stesso, se non intendo cadere in quelle contraddizioni che renderebbero incoerente tutto il mio pensiero e tutto il mio progetto (WEIL, 2003, p. 172).

Si torna a capire perché questo vero e proprio processo di universabilità delle morali storiche e delle massime di ogni singolo individuo, non avrebbe potuto prescindere dalla politica in quanto azione ragionevole e universale sul genere umano ${ }^{34}$. Non perdendo affatto di vista tali premesse, in questo saggio del '70 Weil lo riconobbe a più riprese: si trattava di

\footnotetext{
${ }^{34}$ Questa definizione dell'attività politica come tale era stata elaborata da Weil già nel '56 nella sua Philosophie politique cfr. WEIL, 1973, in particolare p. 14. Si veda anche WEIL, 1961.
} 
qualcosa di «assai démodé, di morale: la fine della storia è la fine della vita immorale» (WEIL, 2003, p. 172). Non può quindi stupire la precisazione immediatamente successiva:

Intendiamoci: non si tratta di imporre agli individui, alle nazioni, alle generazioni una morale positiva o di assicurar loro una felicità che potrebbe non piacergli affatto; non si tratta di reiterare il tentativo di troppi tiranni e di troppi padri tirannici di "rendere felice" il loro popolo e i loro bambini [enfants] (WEIL, 2003, p. 172, i corsivi sono miei) ${ }^{35}$.

È a tal punto che ritorna tutta la prospettiva kantiana di Weil, già sviscerata in relazione all'orientabilità del movimento storico, in virtù dell'interesse della ragione critica:

Non si può "rendere felici". Ciò che tuttalpiù si può fare - in modo alquanto difficile, tant'è che fino ad ora ci si è riusciti solo in minima parte - è permettere la scomparsa delle cause e delle ragioni dei mali, dell'ingiustizia, dell'oppressione, del bisogno materiale, dell'assenza di quella cultura senza la quale nessuno potrebbe concepire semplicemente la felicità; vale a dire un intimo stato di contento e un mondo in cui non si ricercheranno più tutte quelle forme di evasione che, alla lunga, non riusciranno mai a obliare un'insoddisfazione profonda. Ciò a cui mira la morale, per quanto paradossale possa apparire, è di liberarmi alla libertà, alla responsabilità, alla possibilità della mia felicità che, al contempo, possa coesistere con quella di tutti gli altri. In ultima analisi, si tratta della possibilità di trovare un senso alla vita, non in modo arbitrario, ma nei limiti della ragione (WEIL, 2003, p. 173).

La fine della storia: «non c'è nient'altro a cui miri la morale», giacché è quel che «tutta la morale, religiosa, tradizionale, filosofica ha di mira da sempre» (WEIL, 2003, p. 173). In via definitiva, per Weil la fin de l'histoire non poteva che configurarsi come «la fine dell'oppressione che impedisce agli uomini di mantenersi aperti per ciò che è, come diritto dell'umanità, sempre a loro disposizione» (WEIL, 2003, p. 175, i corsivi sono miei). Ciò che la filosofia aveva colto

\footnotetext{
${ }^{35}$ In merito alla prospettiva radicalmente opposta di Kojève, si veda quanto affermato da Domenico Losurdo circa lo "stalinismo" del filosofo russo: «Sbeffeggiato da Trockij quale "piccolo provinciale trasferito per scherzo della storia sul piano dei grandi avvenimenti mondiali", nel 1950 Stalin era assurto, agli occhi di un illustre filosofo (Alexandre Kojève), ad incarnazione dello hegeliano spirito del mondo ed era stato pertanto chiamato a unificare e a dirigere l'umanità, facendo ricorso all'occorrenza a metodi energici e combinando nella sua azione saggezza e tirannide» (LOSURDO, 2019, p. 12). Del resto, è nota (e in tal senso di certo emblematica) la querelle tra Kojève e Leo Strauss sul rapporto tra filosofia e tirannide. Cfr. quindi KOJÈVE, 2004, p. 11-69. Con questo scritto Kojève volle recensire (e criticare) lo studio del "buon liberale" Strauss sul Gerone di Senofonte, intitolato On Tyranny. Cfr. STRAUSS, 2010, pp. 35-147. Cfr. quindi anche la recensione di Kojève a un altro testo di Leo Strauss, Persecution and Art of Writing (Free Press, Glencoe 1952, trad. it. di Giuliano Ferrara e Fiammetta Profili, Scrittura e persecuzione, Marsilio, Venezia 1990), e cioè KOJÈVE, 2004, p. 71-99.
} 
nella categoria della Saggezza - l'ultima categoria della $L F^{36}$ - trovava il suo corrispettivo pratico nell'idea di una comune umanità ancora da realizzare: ecco la vera fin de l'histoire, che poteva essere pensata solo come finalità universale, a un tempo morale e politica, ma non necessaria in sé. La "fine della storia", allora, si ridefiniva a sua volta come il principio regolativo che doveva guidare tanto il processo di liberazione dal bisogno materiale quanto l'universalizzazione dei modi di pensare e di agire dell'uomo ancora violento. La fin de l'histoire, insomma, non poteva più significare assenza totale di conflitti e di contraddizioni o neutralizzazione dell'autenticamente umano. Al contrario, dopo di essa sarebbe stato possibile autodeterminarsi, non essere più degli schiavi costantemente privati di autonomia materiale e morale. Per dirla ancora in altri termini: in un mondo in cui le cause strutturali della violenza prima (quella che aveva rifiutato la Ragione con cognizione di causa) fossero state eliminate, tutti avrebbero potuto decidere davvero della propria vita, nei limiti dell'oggettività della realtà e della ragione:

[...] i mali dell'uomo libero e ragionevole in un mondo libero e ragionevole saranno i suoi propri mali: ancora nella tragedia, sarà lui stesso a portarli a compimento, a portarsi a compimento. Questa, allora, sarà la sua tragedia, non quella delle circostanze, delle condizioni, delle forze esterne. E la lotta tra due sentimenti non sarà più quella tra due posizioni sociali, tra due carriere, tra due interessi o tra due timori ma quella di due individui che avranno scelto la tragedia perché l'avranno preferita agli accomodamenti, ai compromessi, alle compromissioni, al tradimento di se stessi (WEIL, 2003, p. 173 , i corsivi sono miei) ${ }^{37}$.

D’altra parte la filosofia era già riuscita a comprendere tutto ciò: se da un lato è vero, infatti, che noi agiamo «solo per non dover più agire sul piano della storia, di questa storia che abbiamo riconosciuto come quella della violenza e della passione» (WEIL, 2003, p. 174.), dall'altro qui non si trattava «di qualcosa di sconosciuto, di trascendente, d'infinitamente

\footnotetext{
${ }^{36}$ Cfr. $L F$, pp. 589-602.

${ }^{37}$ In buona sostanza, con la "fine della storia" weiliana si sarebbe realizzata la vera autonomia di tutti e di ciascuno: «La fine della storia non significa affatto che nessuno sarà più infelice; essa significa, piuttosto, che l'infelice avrà voluto esserlo e che ogni uomo, alla sola condizione di volerlo (non di desiderarlo o di sognarlo) sarà felice, giacché nulla gli impedirà di volerlo essere» (WEIL, 2003, p. 176). Come ha giustamente sottolineato Pasquale Salvucci lettore di Weil: «Il mondo storico è segnato dai discorsi particolari tra di loro in conflitto. Il mondo umano finalmente realizzato "sarà una società universale senza discorsi particolari" [...] (cfr. $L F$, p. 554, trad. it. modificata), un mondo unificato» (SALVUCCI, 1980, p. 489).
} 
distante, ma di ciò che ciascuno ha conosciuto e conosce nel momento in cui incontra la natura bella, l'arte, la poesia, la vita della presenza dello spirito e del sentimento, l'essere umano nell'amore» (WEIL, 2003, p. 175). È solo così, d'altra parte, che la fine della storia può essere compresa «positivamente, dacché nessuno può prescrivere il contenuto della nostra felicità» (WEIL, 2003, p. 175). Educare alla ricerca del senso, della vera soddisfazione e della libertà di tutti e di ciascuno si confermava la funzione eminentemente pratica della filosofia. Essa, salvaguardando l'autonomia della Ragione, non avrebbe potuto perdere di vista il piano propriamente politico dell'azione ragionevole e universale sul genere umano. Almeno fino a quando la violenza consapevole di sé non fosse stata sconfitta sul suo stesso terreno. Weil lo aveva già scritto nell'ultimo capitolo di Filosofia politica (1956), facendo così intravedere le premesse di fondo per una nuova lettura della fin de l'histoire:

La felicità ragionevole, la felicità che può essere tale per ogni uomo ragionevole (per ogni uomo che non cerca una felicità negata agli altri), diventerà possibilità reale solo se ogni uomo sarà liberato dal bisogno (con la vittoria sulla natura esterna) e dalla passione cieca (con l'educazione sociale e la legge), la propria come quella degli altri. Ma la soddisfazione stessa si incontrerà solo in ciò che non è più azione: essa consiste nella théoria, nella visione di quel senso la cui realtà è presupposta dalla ricerca e dall'azione, da ogni problema e da ogni discorso, anche dal discorso che lo nega. Proprio perché il mondo dell'uomo, il mondo della storia e dell'azione è sensato, l'uomo può cercarvi il senso della sua esistenza - cercarlo e trovarlo, non come si trova un oggetto o un avvenimento o una dottrina già fatta, ma cercarlo e trovarlo nella tensione verso l'universale, verso quella libertà ragionevole che, rifiutando quel che la rifiuta, trova il suo contenuto comprendendosi come visione del tutto, visione che coglie il tutto ma che è pur sempre visione parziale e che deve essersi compiutamente costituita prima di poter comprendere di aver colto quel tutto che una visione affatto diversa aveva già colto, ma da un altro punto di partenza e con un'immagine del tutto differente (WEIL,1973, p. 329) ${ }^{38}$

\footnotetext{
${ }^{38}$ Kant e Aristotele s'incontravano ancora una volta sulla "via posthegeliana" di Weil: in tal caso è il recupero del concetto aristotelico di virtù che non può essere ignorato. Ed è così che la società del genere umano potrà realizzarsi davvero solo se «gli stati storici si mostreranno capaci di oltrepassarsi divenendo, dapprima nella coscienza dei loro cittadini e dei loro governanti, poi nella realtà, ciò che attualmente sono soltanto in sé, cioè delle particolarità morali all'interno della società mondiale, in base al concetto della loro natura, quale può essere dimostrato dalla considerazione filosofica - se sapranno divenire comunità morali libere e riconoscere le necessità del lavoro e dell'organizzazione in vista della realizzazione della ragione, se potranno così dare a tutti gli uomini la possibilità (di cui l'individuo usufruirà o meno) di essere soddisfatti nella dignità della loro libertà ragionevole e concreta, nella vita virtuosa» (WEIL, 1973, 313). A cogliere l'aspetto "aristotelico" della questione, seppur non in questi termini e rimandando implicitamente al concetto "antico" di teoria, è stato ancora Pasquale Salvucci: «Il mondo assolutamente ragionevole si lascia "vedere" da tutti, perché esso è assolutamente limpido, trasparente (non c'è più niente di opaco che ne limiti la "visibilità"). Ora finalmente la ragione "vede" [...], perché interamente
} 
L'unitarietà e la coerenza del discorso "kantiano, post-hegeliano" di Weil risultano così confermate. È certo, comunque, che l'autore non ci restituì affatto un'immagine irenica del suo mondo contemporaneo, lì dove la tensione costante tra pensiero e azione, tra teoria e prassi non poteva che restare sullo sfondo. Ciò che oggi ne possiamo concludere, dunque, è che pur alquanto distanti dalla "fine della storia" weiliana, ripensare il rapporto tra filosofia, politica ed educazione all'universalità riappare, violentemente, come uno dei compiti più impellenti del nostro filosofare. Un compito da cui non sembra più possibile sottrarsi.

\section{Bibliografia}

ARISTOTELE, Etica Nicomachea. Traduzione, introduzione e note di Carlo Natali. Laterza: Bari 2009.

AUFFRET, D. Alexandre Kojève. La philosophie, l'État, la fin de l'histoire. Grasset \& Fasquelle: Paris, 1990.

BARALE, M. Di alcuni usi datati della parola trascendentale e di uno ancora oggi attuale. Giornale di metafisica - Nuova serie, v. XXIX (2007), p. 53-72.

BARALE, M. Ermeneutica e morale. ETS: Pisa, 1988.

BERNARDO, L. M. A. V. Liberdade ou Satisfação: a Crítica de Eric Weil ao Existencialismo. In Jean-Paul Sartre - uma cultura da alteridade. Actas de Colóquio. Universideade Nova de Lisboa, Lisboa. 2005, p. 277-90.

\footnotetext{
"visibile" è il proprio oggetto (la ragione stessa interamente oggettivata). Non vi sono più uomini che vedono per altri, incapaci di vedere. Vivendo all'interno di una struttura interamente conforme a ragione, dove non vi è più lotta per il dominio e il profitto, la soggettività umana può ora realizzare finalmente quello sviluppo onnilaterale delle sue potenzialità che, nel mondo solo in parte ragionevole, essa era in grado di sviluppare soltanto in modo parziale e distorto. Nel mondo della presenza, l'uomo vive felice nella dialettica dei sentimenti ora finalmente liberi, perché non più intaccati dal calcolo e dal profitto» (SALVUCCI, 1980, p. 488-89, nota 35, i corsivi sono miei). Anche la Saggezza, che nella $L F$ aveva ritrovato la Verità (la categoria che, all'inizio, aveva potuto mostrarsi solo attraverso la metafora: cfr. in merito BERNARDO, 2003, p. 120, nota 4 - in cui si richiamano gli studi di Jean Quillien) trova il suo corrispettivo pratico nell'idea di una società del genere umano: a riguardo cfr. TABONI, 1978, p. 135-41, in cui si sviluppa il paragrafo: «Lo Stato mondiale e la saggezza».

È proprio qui, tra l'altro, che è implicato il concetto classico di "vita buona", di cui Weil diede conto in un saggio del '46 indicando, così, quale avrebbe dovuto essere il rapporto tra governanti e governati nella sua contemporaneità: «La grande contraddizione della società moderna, un insieme di tradizione e di rivoluzione, non può essere risolta che dalla parola e dall'azione ragionevoli. Se si vuole che questo grande disegno abbia una qualche possibilità di successo, si devono perseguire a un tempo questi due grandi ideali: una democrazia capace di generare una aristocrazia grazie alla cura di cui essa circonda i meglio dotati; e una democrazia dell'uguaglianza che si sforzi costantemente di innalzare il livello medio, in modo che l'élite non appaia anemica e come sospesa nel vuoto. Gli uni e gli altri devono sapere che vi sono delle verità la cui forma muta nel corso della storia, ma che nessuno ha il diritto di ignorare: lo scopo della società libera, la "vita buona" (vita bona), e le condizioni materiali necessarie per poterle conseguire. Senza la conoscenza un cittadino può essere senz'altro una persona inoffensiva, incapace di fare del male, ma non sarà mai un uomo dabbene (vir bonus)» (WEIL, 1992, p. 54-55).
} 
BERNARDO, L. M. A. V. Linguagem e discurso. Uma hipótese hermenêutica sobre a filosofia de Eric Weil. Imprensa Nacional-Casa da Moeda: Lisboa, 2003.

BIEMEL, W. Die Phänomenologie des Geistes und die Hegel-Renaissance in Frankreich. Stuttgarter HegelTage. Hegel-Studien, Beiheft XI, Bouvier, Bonn 1974, p. 643-655.

CANGUILHEM, G. Hegel en France. Revue d'histoire et de philosophie religieuses, 1948-49, p. 282-297.

CANIVEZ, P. 0 kantismo de Eric Weil. Argumentos, v. 6, n. 11, 2014, p. 9-27.

CAZZANIGA, G. M. L'Azione di Eric Weil. «Presentazione». In: CAZZANIGA, G. M.; LOSURDO, D.; SICHIROLLO, L. (Ed.). Marx e i suoi critici. Quattroventi: Urbino, 1987.

CIAMPA, M. Animali post-storici. In Sulla fine della storia. Liguori: Napoli, 1985.

D’HONT, J. Hegel et l'hegelianisme. Puf: Paris, 1993.

DATI, R. Alexandre Kojève interprete di Hegel. La Città del Sole: Napoli, 1998.

FETSCHER, I. Hegel in Frankreich. Antares, I, n. 3, 1953, p. 3-15.

FILONI, M. Il filosofo della domenica. La vita e il pensiero di Alexandre Kojève. Bollati Boringhieri: Torino, 2008.

FILONI, M. Kojève mon ami. Aragno: Torino, 2013.

FUKUYAMA, F. La fine della storia e l’ultimo uomo. Trad. Delfo Ceni. Bur Rizzoli: Milano, 2017.

GEROULANOS, S. An Atheism that is not humanist. Emerges in French Thought. Stanford University Press: Stanford, 2010.

HEGEL, G. W. F. Enciclopedia delle scienze filosofiche. Trad. B. Croce. Laterza: Bari, 2009.

HYPPOLITE, J. Figures de la pensée philosophique. Puf: Paris, 1971.

JARCZYK, G.; LABARRIÈRE, P.-J. De Kojève à Hegel. 150 ans de pensée hégélienne en France. Albin Michel : Paris, 1996.

KANT, I, Critica della Ragion Pura. Trad. P. Chiodi. Utet: Torino, 1967.

KANT, I. Critica della capacità di giudizio. Trad. L. Amoroso. Rizzoli: Bari, 1995.

KANT, I. Idea per una storia universale dal punto di vista cosmopolitico. In KANT, I. Scritti di Storia, politica e diritto. Trad. F. Gonnelli. Laterza: Roma-Bari, 1995.

KIRSCHER, G. Figures de la violence et de la modernité. Essais sur la philosophie d'Eric Weil. Presses Universitaires de Lille: Lille, 1992.

KOJÈVE, A. Il silenzio della tirannide. Trad. A. Gnoli. Adelphi: Milano, 2004.

KOJÈVE, A. Introduzione alla lettura di Hegel. Lezioni sulla «Fenomenologia dello Spirito» tenute dal 1933 al 1939 all’École Pratique des Hautes Études raccolte e pubblicate da Raymond Queneau. Trad. Gian Franco Frigo. Adelphi, Milano, 1996.

KOJÈVE, A. La metafisica religiosa di Vladimir Solov'ëv. In: KOJÈVE, A. Sostituirsi a Dio. Saggio su Solov'ëv. Cura e introduzione di Marco Filoni. Trad. L. Salvarani. Medusa: Milano 2009.

KOYRÉ, A. Hegel à Jena. In KOYRÉ, A. Études d'histoire de la pensée philosophique. Gallimard: Paris, 1972.

KRÜGER, G. Critique et morale chez Kant. Trad. M. Régnier. Beauchesne: Paris, 1961.

KRÜGER, G. Philosophie und Moral in der Kantischen Kritik. Tübingen: Mohr, 1931.

LOSURDO, D. Stalin. Storia e critica di una leggenda nera. Carocci: Roma, 2019. 
MARX, K. Il capitale. Trad. R. Meyer, a cura di Eugenio Sbardella. Newton: Milano, 1996

NEGRI, A. Hegel nel Novecento. Laterza: Roma-Bari, 1987.

PALMA, M. Foto di Gruppo con servo e signore. Mitologie hegeliane in Koyré, Strauss, Kojève, Bataille, Weil, Queneau. Castelvecchi: Roma, 2017.

PERINE, M. Filosofia e Violência. Sentido e Intenção da Filosofia de Éric Weil. Loyola: São Paulo, 1987.

PIROTTE, D. Alexandre Kojève. Un système anthropologique. Puf: Paris, 2005.

ROTH, M. S. Knowing and History. Appropriations of Hegel in Twentieth-Century France. Cornell University Press: Ithaca and London, 1988.

SALVADORI, R. Hegel in Francia. Filosofia e politica nella cultura francese del Novecento. De Donato: Bari, 1974.

SALVADORI, R. Interpretazioni hegeliane. La nuova Italia: Firenze 1980.

SALVUCCI, P. Il «Kant» di Weil. In: SICHIROLLO, L. (Ed.). Eric Weil. Atti della giornata di studi presso l'Istituto Italiano per gli Studi Filosofici, Napoli, 21 novembre 1987. Quattroventi: Urbino, 1989.

SALVUCCI, P. Politicità della filosofia. Argalìa: Urbino, 1980.

SCHILLER, F. L'educazione estetica. Trad. Giovanna Pinna. Aesthetica: Palermo, 2009.

SICHIROLLO, L. Aristotele. Antropologia, logica, metafisica. In: CIAFRÈ, G.; MORRESI, R.; SICHIROLLO, L.; TABONI, P. F. (Eds.). Filosofia e violenza. Introduzione a Eric Weil. Congedo: Lecce, 1978, p. 93-114.

SICHIROLLO, L. La dialettica degli antichi e dei moderni. Studi su Eric Weil. Il Mulino: Bologna, 1997.

STRAUSS, L. Scrittura e persecuzione. Trad. G. Ferrara e F. Profili. Marsilio: Venezia, 1990.

STRAUSS, L. Sulla tirannide. Trad. D. De Pretto. A cura di Gian Franco Frigo. Adelphi: Milano, 2010.

TABONI, Filosofia politica. Fondamenti e problemi. In: CIAFRÈ, G.; MORRESI, R.; SICHIROLLO, L.; TABONI, P. F. (Eds.). Filosofia e violenza. Introduzione a Eric Weil. Congedo: Lecce, 1978.

TAUBES, J. In diverdente accordo. Scritti su Carl Schmitt. A cura di Elettra Stimilli. Quodlibet: Macerata, 1996.

TOSEL, A. Procès à Marx. Note sur quelques lectures françaises de Marx (1960-1985). In: CAZZANIGA, G. M.; LOSURDO, D.; SICHIROLLO, L. (Ed.). Marx e i suoi critici. Quattroventi: Urbino, 1987.

VALENTINI, F. L'Assoluto nella Logique de la Philosophie. In: SICHIROLLO, L. (Ed.). Eric Weil. Atti della giornata di studi presso l'Istituto Italiano per gli Studi Filosofici, Napoli, 21 novembre 1987. Quattroventi: Urbino, 1989.

VALENTINI, F. La filosofia francese contemporanea. Feltrinelli: Milano, 1958.

VALENTINI. F, Rileggendo la Logica della filosofia. In: VALENTINI, F. Soluzioni Hegeliane. Guerini e Associati: Milano, 2001, pp. 271-343.

VEGETTI, M. La fine della storia. Saggio sul pensiero di Alexandre Kojève. Jaca Book: Milano, 1999.

WEIL, E. Dell'interesse per la storia e altri saggi di filosofia e storia delle idee. Trad. L. Sichirollo. Bibliopolis: Napoli, 1982.

WEIL, E. Essais et conférences 1. Plon : Paris, 1970.

WEIL, E. Filosofia e Politica. Trad. L. Morra. Firenze: Vallecchi, 1965.

WEIL, E. Filosofia politica. Trad. L. Battaglia Cofrancesco. Guida: Napoli, 1973. 
WEIL, E. Hegel e lo Stato. In: WEIL, E. Hegel e lo Stato e altri scritti hegeliani, a cura di Alberto Burgio. Trad. L. Morra. Guerini e Associati: Milano, 1988.

WEIL, E. L'idea di educazione nell'insegnamento americano. In: WEIL, E. Educazione e istruzione. Scienza e discipline umanistiche oggi. Trad. P. F. Taboni. Istituto Italiano per gli Studi Filosofici: Napoli 1992, pp. 54-55.

WEIL, E. Logique de la philosophie. Vrin: Paris, 1950.

WEIL, E. Philosophie et réalité I. Beauchesne: Paris, 2003.

WEIL, E. Philosophie morale. Vrin: Paris, 1961.

WEIL, E. Problemi kantiani. Trad. P. Venditti. Quattroventi: Urbino, 1980.

FUKUYAMA, F.; EATON, G. Francis Fukuyama interview: "Socialism ought to come back". The End of History author on what Karl Marx got right, the rivals to liberal democracy and why he fears a US-China war. (New Statesman, 17 ottobre 2018). Disponível em: https://www.newstatesman.com/culture/observations/2018/10/francis-fukuyama-interview-socialism-ought-come-back. Acesso: $20 / 12 / 2020$.

Recebido em: $21 / 1 / 2021$

Aprovado em: 27/3/2021

\section{Edoardo Raimondi}

Dottore di ricerca (PhD) in Studi Umanistici - Filosofia (SSD M-FIL 06, Ciclo XXXII) presso l'Università degli Studi di Urbino Carlo Bo, con una tesi dal titolo Storia, filosofia e politica alle soglie della postmodernità. Hegel tra Alexandre Kojève ed Eric Weil, sotto la guida del professor Giovanni Bonacina. È inoltre Cultore della materia e borsista di ricerca presso l'Università degli Studi "G. d'Annunzio" di Chieti-Pescara in seno al Dipartimento di Scienze giuridiche e sociali. Ha svolto un periodo di ricerca all'estero presso L'Institut Eric Weil dell'Università di Lille 3. Ha pubblicato per diverse riviste scientifiche, tra cui «Itinerari» (I, 1, Mimesis 2019), «Dianoia» (28, XXIV, 2019) e «Materialismo storico» (1, IV, 2018). Suoi contributi sono apparsi per ETS (in Il Velo scolpito. Dialoghi tra filosofia e letteratura, a cura di Danilo Manca, postfazione di Antonio Prete, Pisa 2013, pp. 105-116) e per Carabba (in Schería. Scritti di antropologia politica. Potere ed esclusione. vol,I, Lanciano 2018, pp. 69-84). 